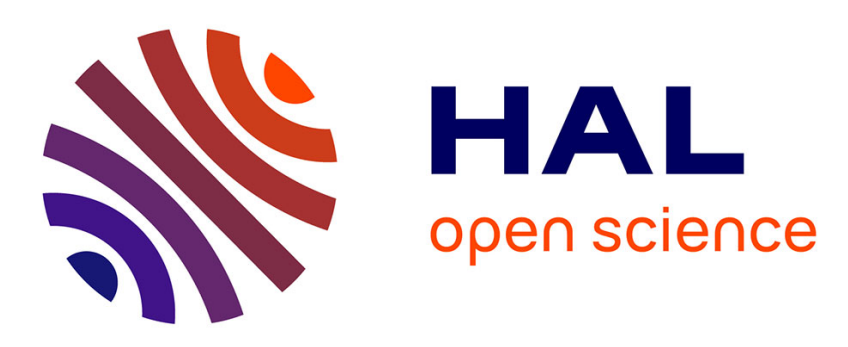

\title{
Evaluation of Network Equivalents for Voltage Optimization in Multi-Area Power Systems
}

Yannick Phulpin, M. Begovic, Marc Petit, Jean-Baptiste Heyberger, Damien Ernst

\section{- To cite this version:}

Yannick Phulpin, M. Begovic, Marc Petit, Jean-Baptiste Heyberger, Damien Ernst. Evaluation of Network Equivalents for Voltage Optimization in Multi-Area Power Systems. IEEE Transactions on Power Systems, 2009, pp.729-743. hal-00411862

\section{HAL Id: hal-00411862}

https://hal-centralesupelec.archives-ouvertes.fr/hal-00411862

Submitted on 31 Aug 2009

HAL is a multi-disciplinary open access archive for the deposit and dissemination of scientific research documents, whether they are published or not. The documents may come from teaching and research institutions in France or abroad, or from public or private research centers.
L'archive ouverte pluridisciplinaire HAL, est destinée au dépôt et à la diffusion de documents scientifiques de niveau recherche, publiés ou non, émanant des établissements d'enseignement et de recherche français ou étrangers, des laboratoires publics ou privés. 


\title{
Evaluation of Network Equivalents for Voltage Optimization in Multi-Area Power Systems
}

\author{
Yannick Phulpin, Student Member, IEEE, Miroslav Begovic, Fellow, IEEE, Marc Petit, \\ Jean-Baptiste Heyberger, and Damien Ernst, Member, IEEE
}

\begin{abstract}
The paper addresses the problem of decentralized optimization for a power system partitioned into several areas controlled by different transmission system operators (TSOs). The optimization variables are the settings for taps, generators' voltages and compensators', and the objective function is either based on the minimization of reactive power support, the minimization of active power losses, or a combination of both criteria. We suppose that each TSO assumes an external network equivalent for its neighboring areas and optimizes without concern for the neighboring systems' objectives its own optimization function. We study, in the context where every TSO adopts the same type of objective function, the performance of an iterative scheme, where every TSO refreshes at each iteration the parameters of its external network equivalents depending on its past internal observations, solves its local optimization problem, and then, applies its "optimal actions" to the power system. In the context of voltage optimization, we find out that this decentralized control scheme can converge to nearly optimal global performance for relatively simple equivalents and simple procedures for fitting their parameters.
\end{abstract}

Index Terms-Decentralized control, multi-TSO power system operation, network equivalent, reactive power scheduling, voltage management.

\section{INTRODUCTION}

I $\mathrm{N}$ multi-area power system operation, it is commonly advocated that new interconnections should be developed, not only for economic reasons but also for operating those systems with higher security margins through sharing of power reserves [1]. However, a better coordination of transmission system operators (TSOs) actions could also help in increasing security margins. Bialek [2] emphasizes that poor coordinated operation may even increase the risk of blackout for multi-TSO power systems.

As discussed in [3], the influence that the controls of one TSO may have on the system variables of its neighboring areas has led, for easing operation, to two major trends for organization and control of interconnected power systems. On one hand, the emergence of some Mega TSOs resulted from the aggregation of several smaller ones (e.g., the regional transmission organization PJM has gradually expanded its operation in the USA

Manuscript received November 16, 2007; revised September 11, 2008. Current version published April 22, 2009. Paper no. TPWRS-00833-2007.

Y. Phulpin and M. Petit are with SUPELEC, Gif sur Yvette 91192, France (e-mail: yannick.phulpin@supelec.fr; marc.petit@supelec.fr).

M. Begovic is with Georgia Institute of Technology, Atlanta, GA 30332 USA (e-mail: miroslav@ece.gatech.edu).

J.-B. Heyberger is with RTE, Versailles 78005, France (e-mail: jean-baptiste. heyberger@rte-france.com).

D. Ernst is with the Belgian National Fund of Scientific Research (FNRS), and also with the University of Liège, Liège B-4000, Belgium (e-mail: dernst@ulg.ac.be).

Digital Object Identifier 10.1109/TPWRS.2009.2016534 over the last years and ensures now the reliability of the electric power supply system in 13 states and the District of Columbia). On the other hand, where the regrouping of TSOs into large entities has not occurred, new strategies to coordinate the actions of those entities have been studied and implemented. By way of example, we mention numerous research papers proposing coordination methods for multi-TSO power systems (e.g., Y. Li et al. outline in [4] a scheme for coordinating path transfers to increase transfer capability while P. Panciatici et al. describe in [5] the benefits of inter-TSO coordination for tertiary voltage control). We also mention the "UCTE Operation Handbook" [6], which sets the rules for close co-operation between member companies to make the best possible use of the benefits offered by interconnected operation.

This paper studies the performance of some decentralized control schemes aiming at solving some optimization problems within such a multi-TSO framework. The study focuses on the optimal reactive power scheduling problem, where the optimality criterion is either based on the minimization of active power losses, the minimization of reactive power support, or the minimization of weighted sums of the active power losses and the reactive power support. Those optimization problems have been widely studied when considering a centralized optimization framework and, in such context, are often referred to as optimal power flow (OPF) problems. Paper [7] addresses the problem of minimization of reactive power support, when a centralized entity has access to all the control variables and has full information about the whole power system. In a similar setting, [8]-[10] consider the problem of minimization of active power losses, and [11], [12] some mixed objective functions. While research in decentralized optimization schemes for power systems is still in its infancy, especially when compared with the large body of work related to centralized optimization, several papers have already studied decentralized optimization schemes in power systems. We mention for example [13], where the authors propose a decentralized OPF that can achieve nearly optimal performance through appropriate exchange of information. In [14], the decentralized scheme studied does not rely on an explicit exchange of information between the TSOs since the information is rather exchanged implicitly by observing the influence of the TSO's actions on the other areas of the power network. Also, M. Ilic et al. highlight in [15] the danger that decentralized optimization may have on power system security when conflicting local strategies result in a reduction of each TSO's own performance criterion.

Hence, there seems to be an overall consensus between academics and industry that decentralized optimization control schemes should exhibit several characteristics such as sim- 


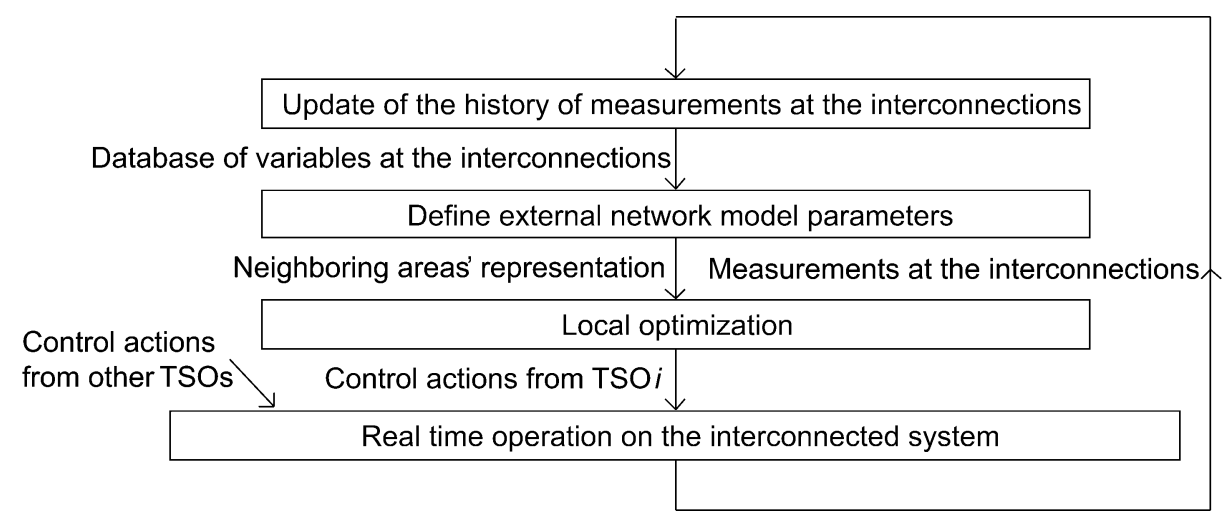

Fig. 1. Role of TSO $i$ in the decentralized optimization scheme.

plicity and robustness with respect to various configurations of the power system and loss of communication channels. They should also give close-to-optimal performance with the optimal performance being achieved by a centralized control scheme having full information about the whole power system and access to every control variable.

We focus on one particular type of the control scheme, whose main features are sketched on Fig. 1. The scheme is iterative in nature and reproduces a decentralized reactive power scheduling. At each iteration, every TSO concurrently solves an optimization problem, for which the function to optimize corresponds to the terms of the global optimization function related to its own area, and then applies its control actions on the system. Also, the TSOs can update, based on the observations made at the interconnections, the parameters of the external network equivalents they use to represent their neighboring areas. The scheme is obviously simple since, among others, it requires no need for communication between the different TSOs or for a centralized authority to coordinate their actions. Two key elements of this iterative control scheme are the type of equivalents used by every TSO to represent its neighboring areas, and the procedure the TSOs adopt to refresh, in every iteration, the parameters of those equivalents to "best" fit the observations made at the interconnections.

The goal of this paper is to study through numerical simulations the influence of those key elements on the performance of the decentralized control scheme, and its robustness in case of several "time-invariant" power systems, where the demand consumption and the active power generation dispatch are fixed. While the set of a priori plausible equivalents may be extremely large, we will mainly consider the cases, where every TSO models its neighboring areas by associating to every interconnection line an equivalent that can be formulated as a set of parametric equality constraints depending only on the current and the voltage at the interconnection. We call those equivalents single-interconnection based equivalents. Similarly, while many mechanisms could be thought of to compute from the observations the parameters of those equivalents, we will limit ourselves to procedures that fit the parameters of an equivalent associated with a particular interconnection to the past measurements of the current and voltage at this interconnection. Also, we will constrain the type of equivalent used as well as the mechanism to fit their parameters to be identical everywhere, regardless of the interconnection or the TSO considered.

By anticipating the conclusions of simulations, we have observed that, in the context of voltage optimization, even with those restrictive assumptions on the set of possible models for representing their neighboring areas, it is possible to formulate decentralized control schemes that perform well, i.e., almost as well as a centralized control scheme. We have organized this paper as follows: in the next section (Section II), we provide a mathematical formulation of the iterative decentralized control scheme, and we detail the centralized control problem, whose solution is the benchmark. In Section III, we detail our analysis method. Section IV reports and analyzes the simulations results for a time-invariant system, while the performance of the decentralized control scheme in a "time-varying" system is outlined in Section V. In Section VI, the computational costs of the scheme are discussed. Finally, some conclusions and directions for future work are drawn in Section VII. An Appendix presents relevant information about the equivalents used in our simulations.

\section{Formulation of the PROBlem}

We start by defining the centralized control problem. Then, we introduce some sets of functional equality constraints that model the network equivalents, and a generic function used to "best" fit the parameters of those equivalents to the observations at the interconnections.

Afterwards, a formulation of the optimization problem faced by a TSO at every instant is proposed. Then, a tabular version of the overall decentralized control scheme is provided, and considerations related to the "quality of an equivalent" are outlined.

We focus in this section on single-interconnection based equivalents. In this case, every interconnection is modeled by an equivalent based on a unique procedure, shared by every TSO, to update its parameters from the history of the measurements of the voltage and the current at the represented interconnection. We will, however, describe in the last paragraph of this section an extension of the decentralized control scheme to the case of the equivalents that cannot be expressed anymore by a set of functional equalities depending on the current and the voltage at only one interconnection.

In the following, we assume that the power system has $n b \mathrm{TSO}$ TSOs referred to as the symbols $1,2, \ldots, n b \mathrm{TSO}$, and that each TSO $i$ has $n b I(i)$ interconnections with its 
neighboring areas. We elected to denote these interconnections by the integer values $1,2, \ldots, n b I(i)$. Also, to simplify the decentralized approach, we assume in this paper that the system is changing slowly enough to be considered "time-invariant." Therefore, we consider a repeating situation, where only the control actions are varying with time. Load consumption and active power generation dispatch are supposed to be constant over the iterations.

\section{A. Centralized Control Problem}

We consider a centralized optimization problem that we compactly write in the form ${ }^{1}$

$$
\min _{\mathbf{u}, \mathbf{I}, \mathbf{V}} C(\mathbf{u}, \mathbf{I}, \mathbf{V})
$$

subject to the inequality and equality constraints

$$
\begin{aligned}
& \mathbf{f}(\mathbf{u}, \mathbf{I}, \mathbf{V}) \leq 0 \\
& \mathbf{g}(\mathbf{u}, \mathbf{I}, \mathbf{V})=0
\end{aligned}
$$

where $\mathbf{u}, \mathbf{I}$, and $\mathbf{V}$ are vectors of control actions, current phasors in each line, and voltage phasors at each bus, respectively. The function $C(\mathbf{u}, \mathbf{I}, \mathbf{V})$ is the objective function of the optimization problem. In this work, we will consider objective functions that can be described by the generic expression $\gamma$ (active_power_losses) $+(1-\gamma)$ (reactive_power_support), where $\gamma \in[0,1]$. Reactive power support is defined as the sum of absolute values of reactive power injected by generators or compensators, and active power losses are defined as the difference between active power injected by generators and absorbed by the loads.

A control action can represent, for example, the tap ratio of a transformer, or a voltage setting for a generator or a compensator. To model a real system, some of those control actions should be of a discrete nature, as in [16], for example. However, as considering discrete and continuous variables would result in mixed-integer nonlinear programming problems that are difficult to address, we have considered only continuous control variables in our simulations. The inequalities (2) represent the constraints on the power system (e.g., transmission line current limits, maximum power production) while the equalities (3) have mainly been introduced to represent power flow equations and economic constraints in terms of active power transfers from one region to another. The variables $\mathbf{I}$ and $\mathbf{V}$ are auxiliary variables, which can be seen as a mathematical tool to formulate the optimization problem in a convenient way. The same procedure will be used later in this section when describing the decentralized control scheme. Such a formulation is widely adopted in the OPF literature, and we refer the reader to the papers [8], [10], [17] for additional information on this subject. We denote by $\left(\mathbf{u}^{*}, \mathbf{I}^{*}, \mathbf{V}^{*}\right)$ a solution of the centralized optimization problem (optimal solution of a union of TSOs).

We assume in this paper that every TSO has the same objective. Therefore, the cost function $C(\mathbf{u}, \mathbf{I}, \mathbf{V})$ can be decomposed into the sum of $n b$ TSO functions $C^{1}(\mathbf{u}, \mathbf{I}, \mathbf{V})+C^{2}(\mathbf{u}, \mathbf{I}, \mathbf{V})+$ $\cdots+C^{n b \operatorname{TSO}}(\mathbf{u}, \mathbf{I}, \mathbf{V})$, where the function $C^{i}$ represents the

${ }^{1}$ Bold fonts are used to highlight vectors of variables and of functions. weighted sum of the active power losses and the reactive power support in the area controlled by TSO $i$. However, this assumption is not a necessity to ensure a good performance of the control scheme. The motivation here is mainly to allow a quantitative evaluation of the performance of the decentralized algorithm in comparison with centralized optimization, which would depend on an arbitrage if the TSOs were to choose different objective formulations.

\section{B. Mathematical Formulation of the Equivalents}

As mentioned in the introduction, this paper addresses only the situations, where, to represent its neighboring areas, every TSO $i$ associates with every interconnection $l$ a set of parametric equality constraints between the current and the voltage at $l$.

We also make additional assumptions as follows:

1) same type of equivalents (or functional form for the equality constraints) is used across all TSOs and across all interconnections;

2) a TSO uses only the past observations of the current and voltage at an interconnection to update the parameters of the equivalent at that interconnection;

3) TSOs use the same mechanism to fit the parameters of the equality constraints to the observations;

4) TSOs measure the value of the current and voltage at the interconnections at some discrete time instants $t$. The time $t$ equal to 0 refers to the first instant at which some measurements are taken. Also, $t+1$ is used to describe the discrete instant following $t$.

Under these assumptions, we can denote by

$$
\mathbf{E}_{\alpha}\left(I^{i, l}, V^{i, l}\right)=0
$$

the set of parametric equality constraints a TSO $i$ associates with the interconnection $l$. The complex variable $I^{i, l}\left(V^{i, l}\right)$ represents the current (voltage) at the interconnection. While the functional form of the multi-dimensional equality constraints is identical, regardless of the $i, l$, or time $t$ at which they are used in the optimization process, they however depend on a vector of parameters $\alpha \in \Omega$ that every TSO $i$ refreshes at every instant for every of its interconnection line $l$.

We define by $\mathbf{H I}_{t}^{i, l}\left(\mathbf{H V}_{t}^{i, l}\right)$ a $(t+1)$-dimensional vector whose components are the values of the current $I^{i, l}$ (voltage $\left.V^{i, l}\right)$ at times $0,1, \ldots, t$. Let $\mathbb{C}$ denote the set of complex numbers, $\mathbb{C}^{n}$ the $n$ th-dimensional space, whose elements are represented by vectors of complex numbers, and $\mathcal{H C}=\cup_{n=1}^{\infty} \mathbb{C}^{2 \times n}$.

With previous assumptions, one can represent the fitting mechanism by a function $\mathbf{F}: \mathcal{H C} \rightarrow \Omega$ such that $\alpha_{t}^{i, l}$, defined as the value of the parameters $\alpha$ that a TSO $i$ associates with its interconnection $l$ and time $t$, is given by

$$
\alpha_{t}^{i, l}=\mathbf{F}\left(\mathbf{H I}_{t}^{i, l}, \mathbf{H V}_{t}^{i, l}\right) .
$$

Appendix A describes several classical equivalents (Thévenin-like, PQ, and PV) and provides for each of them the corresponding set of parametric equality constraints $\mathbf{E}$. The mechanisms $\mathbf{F}$ considered in our simulations for fitting "best" the parameters of the equalities to the observations are also given in this Appendix. 


\section{Decentralized Control Scheme}

Each TSO $i$ is going to assume a "parametric equivalent model" connected to each of its interconnections $l$. First, it will fit the parameters of this model by using the function $\mathbf{F}$, i.e., it will compute the value of $\mathbf{F}\left(\mathbf{H I}_{t}^{i, l}, \mathbf{H V}_{t}^{i, l}\right)$ and set $\alpha_{t}^{i, l}$ equal to the latter value. From there, it can deduce for its interconnection a set of equality constraints $\mathbf{E}_{\alpha=\alpha_{t}^{i, l}}\left(I^{i, l}, V^{i, l}\right)=\mathbf{0}$, where the subscript $\alpha=\alpha_{t}^{i, l}$ has been used in place of ${ }_{\alpha}$ to emphasize that the parameters of the equality constraints have been chosen equal to $\alpha_{t}^{i, l}$. Under the assumption that those $n b I(l)$ sets of equality constraints represent its neighboring areas, the objective function used by TSO $i$ is the weighted sum of active power losses and reactive power support within its own area. We use the notation $\hat{C}^{i}$ to refer to this objective function. The symbol` ${ }^{\wedge}$ reminds us that, since the equivalents do not generally represent perfectly the neighboring areas, the objective function used by TSO $i$ is an approximation of the objective function $C^{i}$ as defined in Section II.A. Similarly, we suppose that the functions $\hat{g}^{i}$ and $\hat{f}^{i}$ refer to the power flow equations and the inequality constraints with areas $i$, respectively. We use also the symbols $\mathbf{u}^{i}, \mathbf{I}^{i}$, and $\mathbf{V}^{i}$ to refer to the controls, currents, and voltages, in TSO $i$ 's area.

Using those notations, the optimization problem TSO $i$ solves at time $t$ is

$$
\min _{\mathbf{u}^{i}, \mathbf{I}^{i}, \mathbf{V}^{i}} \hat{C}^{i}\left(\mathbf{u}^{i}, \mathbf{I}^{i}, \mathbf{V}^{i}\right)
$$

under the inequality and equality constraints

$$
\begin{aligned}
& \hat{\mathbf{f}}^{i}\left(\mathbf{u}^{i}, \mathbf{I}^{i}, \mathbf{V}^{i}\right) \leq \mathbf{0} \\
& \hat{\mathbf{g}}^{i}\left(\mathbf{u}^{i}, \mathbf{I}^{i}, \mathbf{V}^{i}\right)=\mathbf{0} \\
& \mathbf{E}_{\alpha=\alpha_{t}^{i, 1}}\left(I^{i, 1}, V^{i, 1}\right)=\mathbf{0} \\
& \mathbf{E}_{\alpha=\alpha_{t}^{i, 2}}\left(I^{i, 2}, V^{i, 2}\right)=\mathbf{0} \\
& \quad \vdots \\
& \mathbf{E}_{\alpha=\alpha_{t}^{i, n b I(i)}}\left(I^{i, n b I(i)}, V^{i, n b I(i)}\right)=\mathbf{0 .} .
\end{aligned}
$$

\section{Tabular Version of the Decentralized Control Scheme}

We assume that every TSO $i$ computes at the instant $t$ the values of the control actions it will apply to the system at time $t+1$ by solving the optimization problem described by (6)-(11). We denote these values by $\mathbf{u}_{t+1}^{i}$. At the next instant $t+1$, it is assumed that different TSOs measure again the voltages and the currents, both in magnitude and in phase, at their interconnections to update the parameters of their equivalents. At time $t$, the value of the complex current (voltage) at the interconnection $l$ of TSO $i$ is referred to as $I_{t}^{i, l}\left(V_{t}^{i, l}\right)$. This update is realized by adding to the history of the currents $\mathbf{H I}^{i, l}$ (voltages $\mathbf{H V}^{i, l}$ ) the values of $I_{t}^{i, l}\left(V_{t}^{i, l}\right)$. After having updated the histories, the TSOs solve their respective optimization problems, and update their control actions. A tabular version of the exact procedure is given in Fig. 2. It should be emphasized that, to model the effects of the control actions on the evolution of the power system state, we use a power flow algorithm.

\section{1] Set $t=0$. \\ 2] For every $\operatorname{TSO} i$, do the following operations:}

- Measure for every $l=1, \ldots, n b I(i), I_{t}^{i, l}$, and $V_{t}^{i, l}$.

- Update of the history of the measurements with the measurements of current and voltage at every of its interconnections $l$ : $\mathbf{H I}_{t}^{i, l}=\mathbf{H I}_{t-1}^{i, l} \oplus I_{t}^{i, l}$ and $\mathbf{H V}_{t}^{i, l}=$ $\mathbf{H V}_{t-1}^{i, l} \oplus V_{t}^{i, l}$

- Compute for every $l$ the values of $\alpha_{t}^{i, l}=\mathbf{F}\left(\mathbf{H I}_{t}^{i, l}, \mathbf{H V}_{t}^{i, l}\right)$.

- Solve the optimization problem defined by Eqns (6)-(11), and let $\left(\mathbf{u}_{t+1}^{i^{*}}, I_{t+1}^{i^{*}}, V_{t+1}^{i^{*}}\right)$ denote its solution.

3] Use the set $\mathbf{u}_{t+1}$ of different actions $\mathbf{u}_{t+1}^{1^{*}}, \mathbf{u}_{t+1}^{2^{*}}, \ldots$, $\mathbf{u}_{t+1}^{n b T S O^{*}}$ as input of a power flow algorithm. If the power flow algorithm does not converge, return an error message. Otherwise, consider that the bus voltages and line currents computed by the power flow algorithm are equal to the current and voltages measured in $t+1$.

4] Set $t \longleftarrow t+1$, and go back to Step 2 .

Fig. 2. Generic algorithm for simulating the decentralized control scheme. The expression $a=b \oplus c$ sets first the vector $a$ equal to the vector $b$, and then, adds at the end of $a$ the element $c$.

\section{E. Decentralized Control Scheme With More Advanced Equivalents}

In our simulation result section, we will consider two types of equivalents, namely the REI equivalent and the so-called "nonreduced power system" (NPS) equivalent, that give a representation of the power system beyond several interconnections as a single equivalent, instead of one equivalent for each interconnection. To consider those equivalents in our simulations, we have modified the formulation of the optimization problem faced by every TSO $i$ at time $t$ by replacing (9)-(11) by the appropriate equality constraints which, as well as the procedure to update their parameters from the past observations, are given in Appendix B.

\section{SOME CRITERIA FOR ANALYZING THE DECENTRALIZED CONTROL SCHEME}

We study later the influence of specific types of equivalents and fitting procedures on the dynamics induced by the control scheme described in Fig. 2. In the first part of this section, we introduce some criteria to analyze the performance of the control schemes. Afterwards, we illustrate those criteria with some typical dynamics observed in our simulations.

\section{A. Some Criteria for Dynamic Analysis}

For the analysis of the control scheme, we have classified the dynamics into three main classes (singular, convergent, nonconvergent) defined hereafter. For two of these classes, we associate some specific indices that are able to catch in a concise way the relevant information about the suboptimality, the convergence speed, or the "constraint friendliness" of the different schemes.

While one could possibly think about other dynamics that do not naturally fall into any of those classes (e.g., convergence to a limit cycle), we have observed that with the types of equivalents and fitting procedures considered in our simulations, 
the proposed classification was appropriate for all the cases encountered.

1) Singular: We say that the dynamics induced by the control scheme are singular if there exists a value of $t$ for which one (or several) TSO faces an optimization problem with no solution, or for which the action $\mathbf{u}_{t+1}$ leads to a nonconvergent power flow algorithm. We will consider this type of situation unacceptable.

2) Convergent: The process is said to be convergent if there exists a set of control actions $\mathbf{u}_{A}$ such that $\lim _{t \rightarrow \infty}\left\|\mathbf{u}_{t}-\mathbf{u}_{A}\right\| \rightarrow 0$, i.e., the sequence of actions taken by the different TSOs converges towards $\mathbf{u}_{A}$. Note that this situation is only possible because of our assumption of a "time invariant" system.

In such a case, we are interested in knowing for every value of $t$, how far is $\mathbf{u}_{t}$ from $\mathbf{u}^{*}$. By noting that the current and the voltage values outputted by the power flow algorithm run at Step $\mathbf{3}$ of the algorithm depicted in Fig. 2 are functions of $\mathbf{u}_{t}$, one way to define a notion of distance between $\mathbf{u}_{t}$ and $\mathbf{u}^{*}$ would be to compute the difference between $C\left(\mathbf{u}_{t}, \mathbf{I}\left(\mathbf{u}_{t}\right), \mathbf{V}\left(\mathbf{u}_{t}\right)\right)$ and $C\left(\mathbf{u}^{*}, \mathbf{I}\left(\mathbf{u}^{*}\right), \mathbf{V}\left(\mathbf{u}^{*}\right)\right)$. Thus, we define the suboptimality measure $\mathrm{SO}(t)$ as (12) at the bottom of the page.

Although it is certainly informative to study the evolution of $\mathrm{SO}(t)$ with $t$, we found out that, for a convenient analysis of the performance of the control scheme, it was better to focus on two particular indices, deduced from $\mathrm{SO}(t)$. The first one is the asymptotic suboptimality of the scheme, ASO, which is the value of $\mathrm{SO}(t)$ when $t$ tends to infinity $\left(\mathrm{ASO}=\lim _{t \rightarrow \infty} \mathrm{SO}(t)\right)$. The second one is its time to convergence (TC), which we define as the highest value of $t$ for which $\mathrm{SO}(t) \leq 0.9 \times \mathrm{ASO}$ or $1.1 \times \mathrm{ASO} \leq \mathrm{SO}(t)$.

However, those notions of suboptimality and of time to convergence may become inappropriate if the actions $\mathbf{u}_{t}$ do not satisfy the constraints (2), when they are applied to the real power system, which is modeled in this paper by a power flow algorithm (please refer to Step 3 of the algorithm of Fig. 2).

In our simulations, two types of constraints will be considered: limit-type constraints on the reactive power injections and limit-type constraints on the voltages. While running the power flow algorithm with $\mathbf{u}_{t}$ will automatically satisfy the former ones, it may happen that the latter ones will not be. Therefore, we introduce the indices $\mathrm{CV}(t)$, which give the maximum voltage violations at the different instants $t$, and we report its maximum value $\mathrm{CV}_{\max }=\max _{t \in \mathbb{N}} \mathrm{CV}(t)$ and the asymptotic constraint violation $\mathrm{ACV}=\lim _{t \rightarrow \infty} \mathrm{CV}(t)$.

3) Nonconvergent: A process is said to be nonconvergent if it is nonsingular and does not converge. We define several criteria for assessing the performance of this case.

- The average suboptimality AvSO, which is defined as the mean suboptimality over the run of the decentralized optimization algorithm, and its associated standard deviation

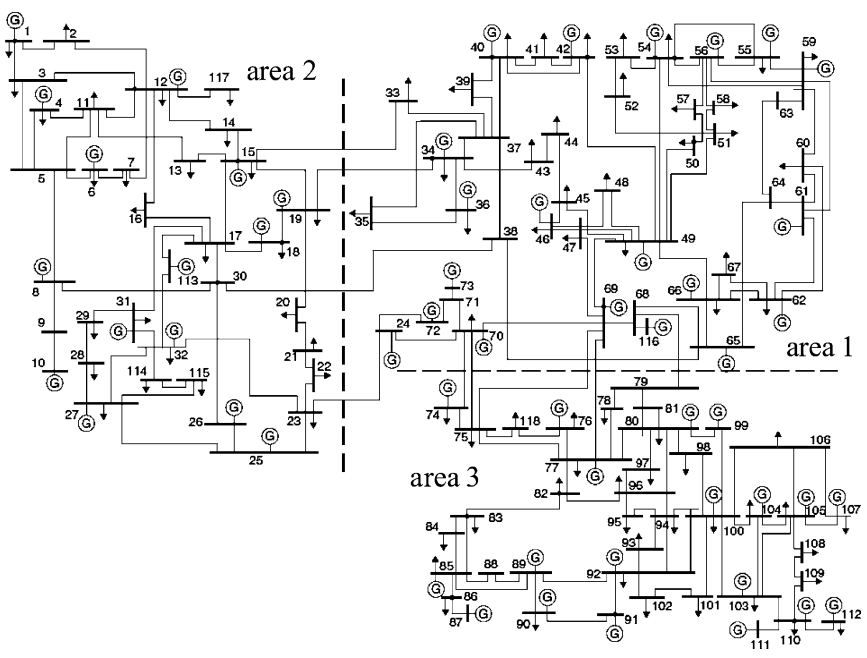

Fig. 3. IEEE 118-bus system with three TSOs.

$\sigma_{\mathrm{AvSO}}$. We note that, by defining in a similar way an AvSO index for a convergent scheme, and by iterating the decentralized control scheme an infinite number of times, we would obtain an AvSO equal to ASO, and a standard deviation $\sigma_{\text {AvSO }}$ equal to 0 .

- The maximum voltage violation $\mathrm{CV}_{\max }$.

- The average voltage violation AvCV defined as the mean voltage violation over the run of the algorithm.

As substitute for $t \rightarrow \infty$, we have used in our simulations a maximum number of iterations equal to 600 . If $\mathrm{SO}(t)$ is not constant over the last iterations, we consider that the case is nonconvergent, although it could, in fact, be convergent or even singular. We however believe that, with such a relatively high limit on the number of iterations, very few cases have been misclassified in our simulation results.

\section{B. Analysis of the Dynamics Through Didactic Examples}

To illustrate the criteria defined in Section III-A, we have chosen to run simulations on the IEEE 118-bus system, whose description can be found in [18]. This system is partitioned into three areas, each of them being independently controlled by a TSO, as depicted in Fig. 3.

In Fig. 4, we report simulation results corresponding to the case, where we minimize reactive power support only $(\gamma=0)$. To model the power system seen beyond a specific interconnection, a Thévenin-like equivalent is used. The parameters of this equivalent are computed by using a fitting procedure based on a weighted least squares (WLS) approach with a weight factor, also called memory factor in this paper, $\beta=0.95$. This memory factor weights past observations with respect to current one (see Section IV for more details). This case is singular since controls

$$
\operatorname{SO}(t)=\frac{C\left(\mathbf{u}_{t}, \mathbf{I}\left(\mathbf{u}_{t}\right), \mathbf{V}\left(\mathbf{u}_{t}\right)\right)-C\left(\mathbf{u}^{*}, \mathbf{I}\left(\mathbf{u}^{*}\right), \mathbf{V}\left(\mathbf{u}^{*}\right)\right)}{C\left(\mathbf{u}^{*}, \mathbf{I}\left(\mathbf{u}^{*}\right), \mathbf{V}\left(\mathbf{u}^{*}\right)\right)}
$$




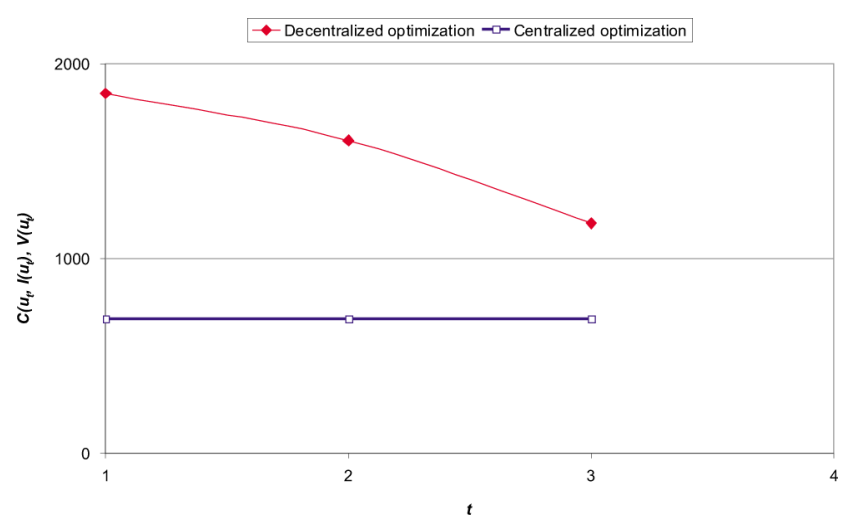

Fig. 4. $C\left(\mathbf{u}_{t}, \mathbf{I}\left(\mathbf{u}_{t}\right), \mathbf{V}\left(\mathbf{u}_{t}\right)\right)$ at each iteration of the decentralized optimization process in the IEEE 118-bus system with three TSOs. The cost function represents reactive power support only. Thévenin-like equivalents are used. The memory factor is chosen equal to 0.95 .
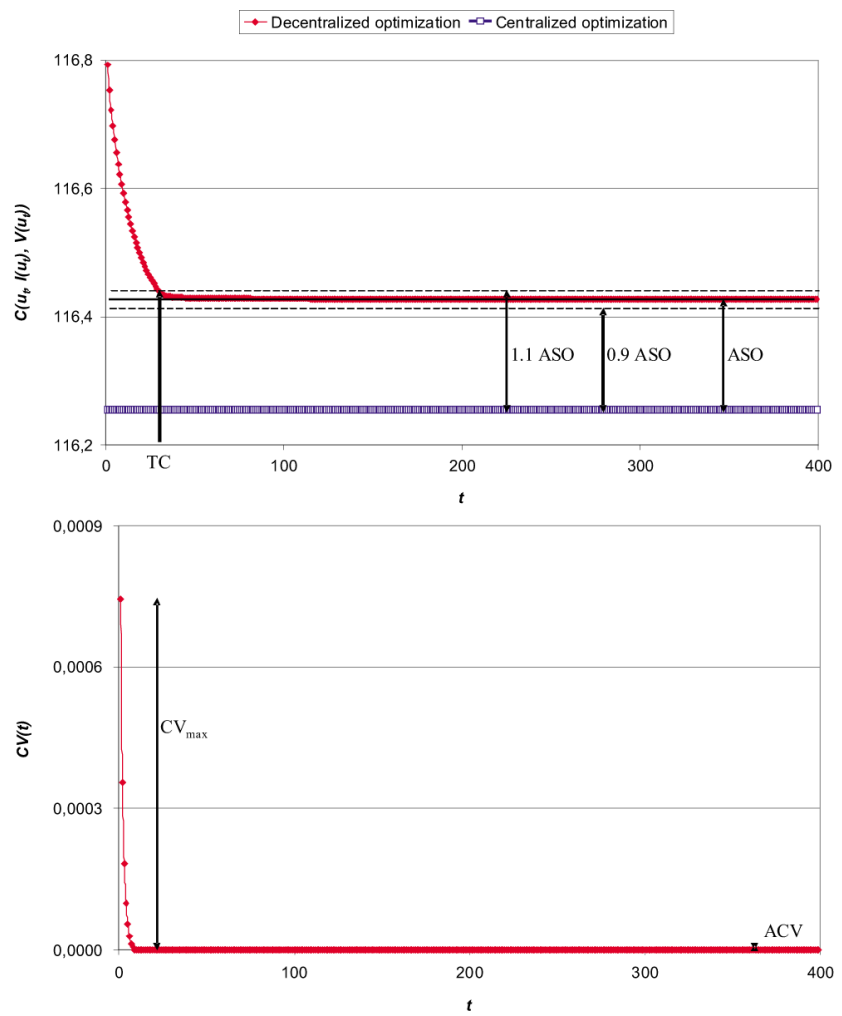

Fig. 5. Decentralized optimization scheme is run on the IEEE 118-bus system with three TSOs. The cost function represents active power losses only. PQ equivalents are used. The memory factor is chosen equal to 0.75 . Top figure represents the time evolution of $C\left(\mathbf{u}_{t}, \mathbf{I}\left(\mathbf{u}_{t}\right), \mathbf{V}\left(\mathbf{u}_{t}\right)\right)$. Bottom figure plots the time evolution of $\mathrm{CV}(t)$.

computed by the TSOs at iteration 3 do not lead to a convergent power flow.

In Fig. 5, we minimize active power losses $(\gamma=1)$. The interconnections are represented by using PQ equivalents together with a WLS based fitting procedure with $\beta=0.75$. For this convergent scheme, the ASO is equal to $0.147 \%$, the time to convergence index (TC) is 29 , the maximum constraint violation $\left(\mathrm{CV}_{\max }\right)$ is 0.0007 p.u., and the asymptotic constraint violation $(\mathrm{ACV})$ is $4 \mathrm{e}-6$ p.u.
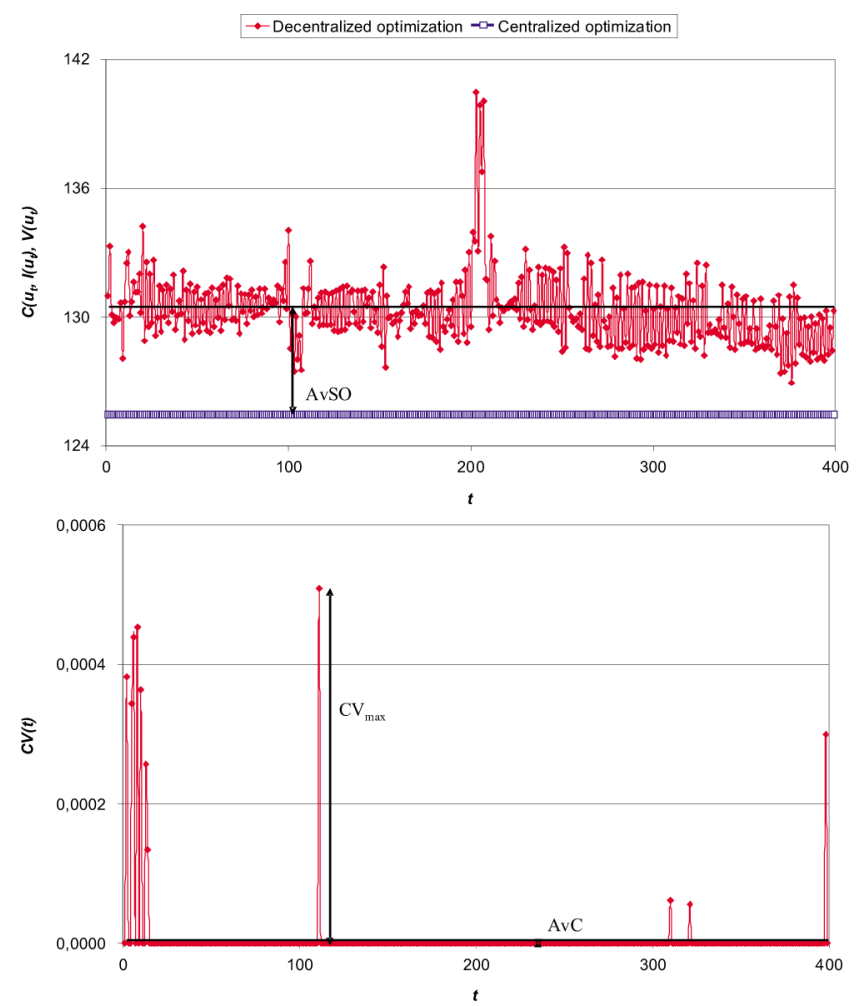

Fig. 6. Decentralized optimization scheme is run on the IEEE 118-bus system with three TSOs. The cost function represents a weighted sum of active power losses and reactive power support $(\gamma=0.97)$. Thévenin-like equivalents are used. The memory factor is chosen equal to 0.95 . Top figure represents the time evolution of $C\left(\mathbf{u}_{t}, \mathbf{I}\left(\mathbf{u}_{t}\right), \mathbf{V}\left(\mathbf{u}_{t}\right)\right)$. The bottom figure plots the time evolution of $\mathrm{CV}(t)$.

In Fig. 6, we minimize a combination of active power losses and reactive power support $(\gamma=0.97)$. The interconnections are represented by using Thévenin-like equivalents together with a WLS based fitting procedure with $\beta=0.95$. This case illustrates a typical nonconvergent behavior, where the ASO is equal to $2.933 \%$ with a standard deviation of $0.77 \%$. Also, the maximum and average constraint violations are 0.0005 p.u. and 8e-6 p.u., respectively.

\section{Simulation Results}

The performance of the decentralized optimization scheme introduced in Section II depends on the type of equivalent used and on the procedure that TSOs adopt to update the parameters of these equivalents. In this section, we study the performance of 15 pairs of "equivalent-fitting procedures" to identify those that systematically lead to high performance. The study considers five types of equivalents and, for each equivalent, three fitting procedures are evaluated. To assess the performance of a specific pair, we have chosen to consider in our study three power systems, six objective functions, and four types of initial conditions (i.e., the state of the system at $t=0$ ). For each pair "equivalent-fitting procedure," this amounts to analyze 72 cases.

The remainder of this section is organized as follows. First we detail the equivalents and fitting functions. Then, we present different cases studied for each pair "equivalent-fitting procedure" and, finally, we discuss the results. 


\section{A. Equivalents and Fitting Functions}

1) Equivalents: We have evaluated the performance of three "single-interconnection based equivalents," namely the PV, Thévenin-like, and PQ equivalents. Their description, together with the set of equality constraints (9)-(11) to which they correspond is given in Appendix A. Also, two more advanced equivalents, namely the REI equivalent and the NPS, have been considered. Their description and mathematical formulation are provided in Appendix B.

2) Fitting Functions: We have used in our simulations fitting functions based on a weighted least squares approach. We refer the reader to Appendix A.D for a detailed description of this approach. Fitting procedures differ from one another by the value of a memory (or weight) factor $\beta \in[0,1]$. A past observation obtained at instant $k$ is weighted with respect to the current one obtained at instant $t$ by a factor $\beta^{t-k}$. We have considered the following values of $\beta$ in our simulations:

- $\beta=0.5$ (low memory factor);

- $\beta=0.75$ (medium memory factor);

- $\beta=0.95$ (high memory factor).

Values of $\beta=0$ and $\beta=1$ have not been considered on purpose. Indeed, if we consider $\beta=0$ - that is, only the last measurements are taken into account - then, the solution of the WLS problem is undefined for the REI and the Thévenin-like equivalents. On the other hand, with a value of $\beta=1$, the function to be minimized by the WLS approach can not be bounded anymore for any value of $t$, which is the reason why we elect not to consider this case.

\section{B. Conditions of Simulations}

1) Benchmarks: Three power system benchmarks have been considered, namely:

- the IEEE 39 bus New England system with three different TSOs introduced in [14];

- the IEEE 118-bus system with two different TSOs, which is used in [19];

- the IEEE 118-bus system with three different TSOs, which is derived from the two TSO IEEE 118-bus system and is represented in Fig. 3.

Their description can be found in the Matpower data set [18]. When the separation between two areas happens in between two buses, two fictitious buses are added at each side of the interconnection.

2) Optimization Problems: Six optimization functions have been chosen. All of them can be written under the generic form $\gamma($ active_power_losses $)+(1-\gamma)$ (reactive_power_support) and differ from each other by the value of $\gamma \in[0,1]$. The six different values of $\gamma$ are: 0, 0.9, 0.94, 0.97, 0.99, or 1 .

Control variables are generator and compensator voltages, and tap settings. The problem is constrained by maximum and minimum values for bus voltages, generators' and compensators' reactive power injections, and tap settings. Also, additional constraints have been introduced to maintain constant active power exchanges between the areas.

3) Initial State: The state of the system at time $t=0$ will influence the outcome of the first optimization problem solved by the TSO and, subsequently, every action $\mathbf{u}_{t}$ since $\mathbf{u}_{t}$ is function of $\mathbf{u}_{0}, \mathbf{u}_{1}, \ldots, \mathbf{u}_{t-1}$. The influence of four types of initial states is studied.

- Centrally-optimized state: The initial state is the solution of the centralized optimization problem defined by (1)-(3).

- High-voltage state: The initial state is the solution of an optimization problem, whose objective is to maximize of the average bus voltage, while ensuring that every voltage is below its upper limit value.

- Low-voltage state: The initial state corresponds to the minimum average bus voltage.

- Medium-voltage state: The initial state is the solution of an optimization problem, whose objective is to bring the average bus voltage as close as possible to 1 p.u.

\section{Results}

1) Average Performance of Each Pair "Equivalent-Fitting Function": To analyze the average performance of every pair "equivalent-fitting function" over the 72 simulation cases, we have reported in Table I the frequency of each type of dynamics (singular, convergent, nonconvergent), and the average values of the different indices introduced in Section III.

As one can note, singular dynamics are only induced by REI and Thévenin-like equivalents. For those singular cases, we have observed that they are most often corresponding to the absence of a power flow solution after several iterations. The REI and Thévenin-like equivalents are the only ones that set voltage angle differences between interconnections. We found out that those voltage angle differences "predicted" at time $t$ by the fitting procedure are significantly different from those that can be measured at time $t+1$, when local controls $\mathbf{u}_{t+1}^{1}, \mathbf{u}_{t+1}^{2}, \ldots, \mathbf{u}_{t+1}^{n b T S O}$ are applied to the power system. This strong difference is in our opinion the root cause for the singularity induced by the REI and Thévenin-like equivalents. To avoid singular control schemes, we will qualify Thévenin-like and REI equivalent as not appropriate for use with our decentralized control scheme, and therefore, we will not further discuss their performance.

The dynamics induced by the PV, PQ, and NPS equivalents can be either convergent or nonconvergent. Convergence is almost always observed with NPS and PQ equivalents, and in almost $40 \%$ of the cases with PV equivalent. We have observed a low influence of $\beta$ on the classification of dynamics. Also, with the investigated values, we found out that increasing $\beta$ could either increase or decrease the amount of convergent cases.

In terms of suboptimality, the PQ equivalent offers the best performance with a low average suboptimality of around $5 \%$. It is followed by the PV equivalent, for which the average suboptimality is less than $9 \%$ for nonconvergent cases and less than $13.6 \%$ for convergent ones. Finally, the NPS equivalent leads to the highest suboptimalities, which are in average more than five times higher than with PQ equivalent. This result can be explained by the fact that the competition between different TSOs is stronger when they have more accurate information on other TSOs' network and past actions. The stronger competition leads here to local optimization relying more on external TSOs' control actions, which results, after several iterations, in a highly suboptimal state. 
TABLE I

Analysis of the Dynamics Induced by 15 Pairs "Equivalent-FitTing Function." For Each Pair, 72 Cases ARe Studied (Three Power Systems $\times$ Six Objective Functions $\times$ Four Initial States). The Repartition in Dynamics' Classes AND THE AVERAGE VALUE OF THE INDICES INTRODUCED IN SECTION III ARE REPORTED

\begin{tabular}{|c|c|c|c|c|c|c|c|c|c|c|c|c|c|}
\hline \multicolumn{2}{|c|}{ Equiv.-Fit. funct. } & \multicolumn{3}{|c|}{ Classification } & \multicolumn{4}{|c|}{ Convergent cases } & \multicolumn{5}{|c|}{ Non-convergent cases } \\
\hline Equiv. & $\beta$ & Sing. & Conv. & Non-conv. & $A S O$ & $T C$ & $C V_{\max }$ & $A C V$ & $A v S O$ & $\sigma_{A v S O}$ & $T C$ & $C V_{\max }$ & $A c C V$ \\
\hline PV & 0.50 & $0 \%$ & $43 \%$ & $57 \%$ & $13.6 \%$ & 40 & 0.0018 & 0.0003 & $8.5 \%$ & $1.6 \%$ & 565 & 0.0007 & 0.0003 \\
\hline $\mathrm{PV}$ & 0.75 & $0 \%$ & $40 \%$ & $60 \%$ & $13.8 \%$ & 44 & 0.0023 & 0.0002 & $7.5 \%$ & $1.4 \%$ & 563 & 0.0006 & 0.0002 \\
\hline PV & 0.95 & $0 \%$ & $33 \%$ & $67 \%$ & $14.3 \%$ & 29 & 0.0019 & 0.0001 & $6.4 \%$ & $1.1 \%$ & 568 & 0.0010 & 0.0002 \\
\hline Thev. & 0.50 & $89 \%$ & $0 \%$ & $11 \%$ & & & & & $0.3 \%$ & $0.2 \%$ & 600 & 0.0041 & 0.0008 \\
\hline Thev. & 0.75 & $79 \%$ & $0 \%$ & $21 \%$ & & & & & $2.6 \%$ & $1.0 \%$ & 600 & 0.0084 & 0.0004 \\
\hline Thev. & 0.95 & $54 \%$ & $0 \%$ & $46 \%$ & & & & & $4.6 \%$ & $1.3 \%$ & 598 & 0.0103 & 0.0003 \\
\hline $\mathrm{PQ}$ & 0.50 & $0 \%$ & $83 \%$ & $17 \%$ & $4.3 \%$ & 12 & 0.0003 & 0.0000 & $5.6 \%$ & $0.9 \%$ & 456 & 0.0005 & 0.0000 \\
\hline $\mathrm{PQ}$ & 0.75 & $0 \%$ & $92 \%$ & $8 \%$ & $3.9 \%$ & 15 & 0.0003 & 0.0000 & $2.1 \%$ & $0.5 \%$ & 21 & 0.0006 & 0.0000 \\
\hline PQ & 0.95 & $0 \%$ & $92 \%$ & $8 \%$ & $3.9 \%$ & 45 & 0.0004 & 0.0000 & $2.1 \%$ & $0.5 \%$ & 347 & 0.0009 & 0.0001 \\
\hline REI & 0.50 & $100 \%$ & $0 \%$ & $0 \%$ & & & & & & & & & \\
\hline REI & 0.75 & $100 \%$ & $0 \%$ & $0 \%$ & & & & & & & & & \\
\hline REI & 0.95 & $100 \%$ & $0 \%$ & $0 \%$ & & & & & & & & & \\
\hline NPS & 0.50 & $0 \%$ & $100 \%$ & $0 \%$ & $36.7 \%$ & 2 & 0.0003 & 0.0003 & & & & & \\
\hline NPS & 0.75 & $0 \%$ & $100 \%$ & $0 \%$ & $21.7 \%$ & 3 & 0.0003 & 0.0003 & & & & & \\
\hline NPS & 0.95 & $0 \%$ & $93 \%$ & $7 \%$ & $21.4 \%$ & 6 & 0.0003 & 0.0003 & $2.0 \%$ & $0.7 \%$ & 600 & 0.0001 & 0,0000 \\
\hline
\end{tabular}

TABLE II

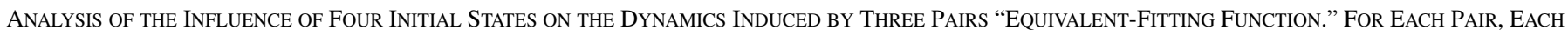

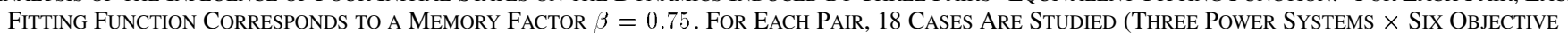
Functions). The RePartition in Dynamics' Classes and the Average Value of tHe INDices Introduced IN SECTION III ARE REPORTED

\begin{tabular}{|c||c||c|c|c||c|c|c||c|c||c|c|c|c|}
\hline \multicolumn{1}{|c||}{} & \multicolumn{1}{|c||}{} & \multicolumn{3}{|c||}{ Classification } & \multicolumn{4}{c||}{ Convergent cases } & \multicolumn{4}{c|}{ Non-convergent cases } \\
\hline Equiv. & Initial Data & Sing. & Conv. & Non-conv. & $A S O$ & $T C$ & $C V_{\max }$ & $A C V$ & $A v S O$ & $\sigma_{A v S O}$ & $T C$ & $C V_{\max }$ & $A v C V$ \\
\hline PV & CO & $0 \%$ & $17 \%$ & $83 \%$ & $0.2 \%$ & 2 & 0.0014 & 0.0006 & $8.3 \%$ & $1.6 \%$ & 600 & 0.0004 & 0.0001 \\
\hline PV & Low & $0 \%$ & $67 \%$ & $33 \%$ & $18.6 \%$ & 44 & 0.0034 & 0.0001 & $6.7 \%$ & $1.2 \%$ & 509 & 0.0020 & 0.0002 \\
\hline PV & High & $0 \%$ & $11 \%$ & $89 \%$ & $0.3 \%$ & 22 & 0.0000 & 0.0000 & $7.7 \%$ & $1.5 \%$ & 564 & 0.0006 & 0.0002 \\
\hline PV & Medium & $0 \%$ & $67 \%$ & $33 \%$ & $14.8 \%$ & 58 & 0.0017 & 0.0003 & $5.9 \%$ & $0.8 \%$ & 524 & 0.0000 & 0.0000 \\
\hline PQ & CO & $0 \%$ & $94 \%$ & $6 \%$ & $0.3 \%$ & 14 & 0.0001 & 0.0000 & $1.7 \%$ & $0.2 \%$ & 27 & 0.0002 & 0.0000 \\
\hline PQ & Low & $0 \%$ & $89 \%$ & $11 \%$ & $14.7 \%$ & 17 & 0.0008 & 0.0000 & $2.3 \%$ & $0.3 \%$ & 33 & 0.0016 & 0.0001 \\
\hline PQ & High & $0 \%$ & $94 \%$ & $6 \%$ & $0.6 \%$ & 12 & 0.0003 & 0.0000 & $3.8 \%$ & $1.1 \%$ & 7 & 0.0000 & 0.0000 \\
\hline PQ & Medium & $0 \%$ & $89 \%$ & $11 \%$ & $0.4 \%$ & 16 & 0.0002 & 0.0000 & $1.3 \%$ & $0.5 \%$ & 13 & 0.0003 & 0.0000 \\
\hline NPS & CO & $0 \%$ & $100 \%$ & $0 \%$ & $21.7 \%$ & 5 & 0.0003 & 0.0003 & & & & & \\
\hline NPS & Low & $0 \%$ & $100 \%$ & $0 \%$ & $21.7 \%$ & 2 & 0.0003 & 0.0003 & & & & & \\
\hline NPS & High & $0 \%$ & $100 \%$ & $0 \%$ & $21.7 \%$ & 2 & 0.0003 & 0.0003 & & & & & \\
\hline NPS & Medium & $0 \%$ & $100 \%$ & $0 \%$ & $21.7 \%$ & 2 & 0.0003 & 0.0003 & & & & \\
\hline
\end{tabular}

As for the "constraint friendliness" associated with different types of equivalents, it is remarkable to observe that, even if the TSOs choose actions only by considering their own constraints, violations induced by the decentralized control scheme are negligible.

The parameter $\beta$, which weights long term measurements with respect to short-term ones, was not found to strongly influence the performance of the different schemes, at least for the values under consideration. However, we have found that for PQ equivalent, which offers the best performance, large values of $\beta$ were leading to more convergent cases, even if the time to convergence was increasing with $\beta$. In the rest of this section, we will only consider a single value of $\beta$ equal to 0.75 .

2) Impact of the Initial State: Table II represents collection of mean values of the main evaluation indices for PV, PQ, and NPS equivalents with a memory factor $\beta=0.75$. Every benchmark power system and optimization function has been considered when computing those values.

As one can observe, regardless of the initial state, the NPS equivalent leads to a convergent scheme, and gives the same mean value for ASO. We have actually observed convergence towards the same vector $\mathbf{u}$ with every initial state.

A different behavior has been observed when considering control schemes based on PQ equivalents. In such cases, we found out that the further the initial state was from the centrally-optimized one, the higher the suboptimality of the scheme tended to be. Indeed, by starting from the centrally-optimized state, we have an average value for ASO equal to $0.3 \%$ while it is equal to $14.7 \%$ when using a low-voltage initial state, which, in the set of initial states under consideration, is the most suboptimal. 
TABLE III

Analysis of the Dynamics ObTained in Three Power Systems While Using Three Different Equivalents, a Memory Factor $\beta=0.75$, and a Medium-Voltage Initial State. For Each Case, Six Objective Functions Are Considered

\begin{tabular}{|c|c|c|c|c|c|c|c|c|c|c|c|c|c|}
\hline & & \multicolumn{3}{|c|}{ Classification } & \multicolumn{4}{|c|}{ Convergent cases } & \multicolumn{5}{|c|}{ Non-convergent cases } \\
\hline Equiv. & bus/TSO & Sing. & Conv. & Non-conv. & $A S O$ & $T C$ & $C V_{\max }$ & $A C V$ & $A v S O$ & $\sigma_{A v S O}$ & $T C$ & $C V_{\max }$ & $A v C V$ \\
\hline $\mathrm{PV}$ & $39 / 3$ & $0 \%$ & $83 \%$ & $17 \%$ & $21.7 \%$ & 70 & 0.0018 & 0.0000 & $16.6 \%$ & $2.1 \%$ & 600 & 0.0001 & 0.0000 \\
\hline PV & $118 / 2$ & $0 \%$ & $17 \%$ & $83 \%$ & $0.1 \%$ & 102 & 0.0011 & 0.0000 & $3.8 \%$ & $0.5 \%$ & 508 & 0.0000 & 0.0000 \\
\hline PV & $118 / 3$ & $0 \%$ & $100 \%$ & $0 \%$ & $11.5 \%$ & 40 & 0.0017 & 0.0006 & & & & & \\
\hline PQ & $39 / 3$ & $0 \%$ & $100 \%$ & $0 \%$ & $0.0 \%$ & 30 & 0.0004 & 0.0000 & & & & & \\
\hline PQ & $118 / 2$ & $0 \%$ & $100 \%$ & $0 \%$ & $0.3 \%$ & 9 & 0.0001 & 0.0000 & & & & & \\
\hline $\mathrm{PQ}$ & $118 / 3$ & $0 \%$ & $67 \%$ & $33 \%$ & $1.2 \%$ & 6 & 0.0000 & 0.0000 & $1.3 \%$ & $0.5 \%$ & 13 & 0.0003 & 0.0000 \\
\hline NPS & $39 / 3$ & $0 \%$ & $100 \%$ & $0 \%$ & $4.8 \%$ & 2 & 0.0007 & 0.0007 & & & & & \\
\hline NPS & $118 / 2$ & $0 \%$ & $100 \%$ & $0 \%$ & $51.8 \%$ & 2 & 0.0000 & 0.0000 & & & & & \\
\hline NPS & $118 / 3$ & $0 \%$ & $100 \%$ & $0 \%$ & $8.6 \%$ & 2 & 0.0002 & 0.0002 & & & & & \\
\hline
\end{tabular}

TABLE IV

Analysis of the Dynamics Obtained With Six Objective Functions While Using Three Different Equivalents, a MEMory Factor $\beta=0.75$, AND A Medium-Voltage InItIAL State In THE IEEE 118-Bus System With Three TSOs

\begin{tabular}{|c|c|c|c|c|c|c|c|c|c|c|c|c|c|}
\hline \multirow[b]{2}{*}{ Equiv. } & \multirow[b]{2}{*}{$\gamma$} & \multicolumn{3}{|c|}{ Dynamics } & \multicolumn{4}{|c|}{ Convergent cases } & \multicolumn{5}{|c|}{ Non-convergent cases } \\
\hline & & Sing. & Conv. & Non-conv. & $A S O$ & $T C$ & $C V_{\max }$ & $A C V$ & $A v S O$ & $\sigma_{A v S O}$ & $T C$ & $C V_{\max }$ & $A v C V$ \\
\hline PV & 0.00 & 0 & 1 & 0 & $27.8 \%$ & 12 & 0.0021 & 0.0000 & & & & & \\
\hline $\mathrm{PV}$ & 0.90 & 0 & 1 & 0 & $15.8 \%$ & 24 & 0.0011 & 0.0000 & & & & & \\
\hline $\mathrm{PV}$ & 0.94 & 0 & 1 & 0 & $12.6 \%$ & 26 & 0.0010 & 0.0000 & & & & & \\
\hline $\mathrm{PV}$ & 0.97 & 0 & 1 & 0 & $9.9 \%$ & 29 & 0.0006 & 0.0000 & & & & & \\
\hline PV & 0.99 & 0 & 1 & 0 & $2.6 \%$ & 43 & 0.0025 & 0.0022 & & & & & \\
\hline $\mathrm{PV}$ & 1.00 & 0 & 1 & 0 & $0.1 \%$ & 106 & 0.0030 & 0.0017 & & & & & \\
\hline PQ & 0.00 & 0 & 0 & 1 & & & & & $2.3 \%$ & $0.9 \%$ & 6 & 0.0000 & 0.0000 \\
\hline PQ & 0.90 & 0 & 1 & 0 & $1.3 \%$ & 9 & 0.0000 & 0.0000 & & & & & \\
\hline PQ & 0.94 & 0 & 1 & 0 & $1.2 \%$ & 9 & 0.0000 & 0.0000 & & & & & \\
\hline PQ & 0.97 & 0 & 1 & 0 & $1.3 \%$ & 3 & 0.0000 & 0.0000 & & & & & \\
\hline PQ & 0.99 & 0 & 1 & 0 & $0.9 \%$ & 2 & 0.0000 & 0.0000 & & & & & \\
\hline PQ & 1.00 & 0 & 0 & 1 & & & & & $0.2 \%$ & $0.0 \%$ & 20 & 0.0005 & 0.0000 \\
\hline NPS & 0.00 & 0 & 1 & 0 & $26.8 \%$ & 2 & 0.0002 & 0.0002 & & & & & \\
\hline NPS & 0.90 & 0 & 1 & 0 & $9.8 \%$ & 2 & 0.0002 & 0.0002 & & & & & \\
\hline NPS & 0.94 & 0 & 1 & 0 & $6.5 \%$ & 2 & 0.0002 & 0.0002 & & & & & \\
\hline NPS & 0.97 & 0 & 1 & 0 & $4.0 \%$ & 2 & 0.0002 & 0.0002 & & & & & \\
\hline NPS & 0.99 & 0 & 1 & 0 & $2.5 \%$ & 2 & 0.0002 & 0.0002 & & & & & \\
\hline NPS & 1.00 & 0 & 1 & 0 & $2.0 \%$ & 2 & 0.0002 & 0.0002 & & & & & \\
\hline
\end{tabular}

With PV equivalents, the initial state strongly impacts the classification of the dynamics: the percentage of convergent cases amounts $67 \%$ with a low-voltage or a medium-voltage initial state, whereas less than $17 \%$ of the cases are convergent when using a high-voltage or centrally-optimized (CO) initial state. Actually, when using a value of $\gamma \leq 0.99$ with a high-voltage or a $\mathrm{CO}$ initial state, the decentralized control scheme using PV equivalents never leads to a convergent result. As with PQ equivalents, one can observe that starting from a low-voltage initial state induces a much higher average value for the ASO index than when starting from a $\mathrm{CO}$ or a high-voltage state. However, we should stress here that comparing those average values of ASO is not meaningful since they have been computed for different cases. Indeed, for the CO (high-voltage) initial state, the average is computed for three (two) cases that correspond to an objective function for which $\gamma=1$, while for the low-voltage initial state, nine additional cases are considered in the average computation. Actually, the average values of ASO corresponding to the same cases do not show strong dependence on the initial state.

3) Impact of the Power System: Table III illustrates the influence of the power system on the dynamics induced by the control scheme. As one can observe, the dynamics may strongly depend on the power system. We found that the performance of the scheme is dependent on the ratio between number of interconnections divided by the size of the power system, especially when considering $\mathrm{PQ}$ and $\mathrm{PV}$ equivalents. The higher that ratio is, the less performing the scheme tends to be.

4) Impact of the Optimization Function: Table IV studies the influence of the optimization functions on the dynamics of the decentralized control scheme. One can observe that the choice of the equivalents does not have much influence when the objective function is the minimization of active power losses. It is obvious that TSOs try, in this context, to maximize the voltage level across the area they are responsible for, and thus there is no important competition around interconnections. However, when 
$\gamma$ is lower than 1, the objective function is more conflicting, and the type of the equivalent is critical. Indeed, in such cases, the TSOs acting in a greedy way are tempted to lower their voltages so as to maximize the reactive power import from other TSOs. This leads over the iterations to a gradual decrease of the voltage profile and, in return, to a highly suboptimal system state. We note that this phenomenon is particularly important when using NPS equivalents.

We observe, however, very promising results with PQ equivalents: the asymptotic suboptimality is smaller than $2.4 \%$ for any value of $\gamma$ and every benchmark considered. On the contrary, PV equivalents are performing less well: the asymptotic suboptimality indeed increases with the weight of reactive power support for any benchmark. Nevertheless, both types of equivalents tend to lead to higher performance than those observed with NPS equivalent, which leads to a higher competition between different TSOs.

\section{ApPlication to a “Time-VArying” System}

In the previous section, it has been assumed, mostly for the sake of simplicity, that the load consumption, generation dispatch and network topology remain constant from one iteration to another. With such an assumption, it has been shown that the performance of the decentralized control scheme with PQ equivalents is nearly optimal. Before adopting such a scheme for implementation in a real power system, it is important to study whether it would perform well in "time-varying" systems.

The relatively fast convergence properties of the control scheme shown in the previous section suggest that such an application could be feasible. Indeed, suppose that at one instant, a sudden change of topology occurs (e.g., loss of a transmission circuit). Then, due to its fast convergence properties, the scheme should converge again within a few iterations to a new operating point corresponding to a nearly optimal solution of the new configuration of the system. Moreover, if the time between two iterations is small, the transient period during which the system operates in higher suboptimal conditions would be short. However, it is important to mention that, as shown in previous section (and more particularly in Table I), the time to convergence is highly dependent on the memory factor $\beta$, which weights past observations with respect to the current ones in the parameter fitting procedure. Roughly speaking, in the case of PQ equivalents, the higher the value of $\beta$, the better the quality of the convergence point but, at the same time, the time to convergence will be larger. In the case of a "time-varying" system, tuning the value of the parameter $\beta$ is important to address the tradeoff between the adaptivity of the scheme and efficiency of the values of control variables that it tends to converge to.

To illustrate the performance of the decentralized control scheme in a "time-varying" system, we have chosen to report simulation results obtained for one of the test systems, the IEEE 118-bus system partitioned into three areas. We consider a time varying load profile over a five day period, where the delay between two discrete instants $t$ and $t+1$ is equal to thirty minutes. This delay has been chosen relatively long on purpose since inaccuracy related to the time-varying aspects

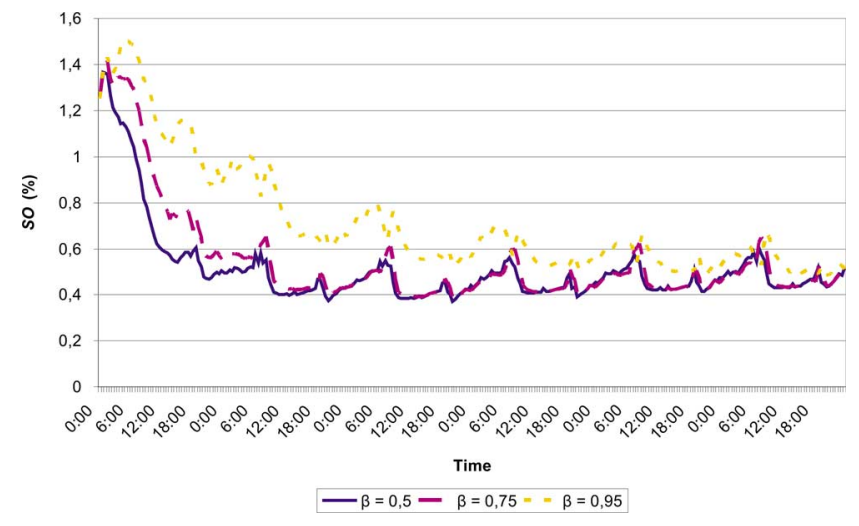

Fig. 7. Evolution of the suboptimality index $S O$ for a time-varying IEEE 118-bus system with three TSOs. Every TSO is assumed to minimize active power losses in its own area.

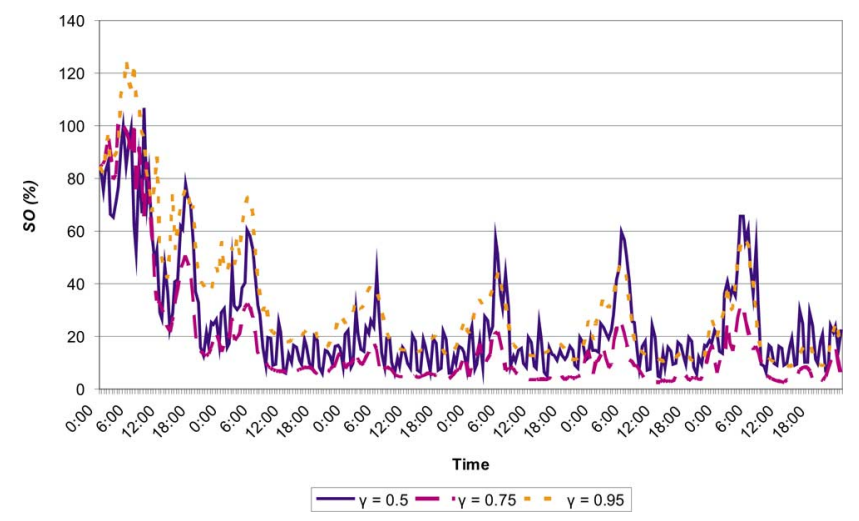

Fig. 8. Evolution of the suboptimality index $S O$ for a time-varying IEEE 118-bus system with three TSOs. Every TSO is assumed to minimize reactive power support in its own area.

of the model would be almost negligible with a shorter delay (say, $5 \mathrm{~min}$ instead of $30 \mathrm{~min}$ ), which would reduce the interest for those simulations as far as the investigation of the impact of time-varying model is concerned. The time varying load profile has been established by reproducing the load consumption observed in the French system during the period January 17-21, 2007. More specifically, we have averaged the French consumption over this period, computed the ratios $r(t)$ between the consumption at different instants $t$ and this average, and then, considered that the load profile at instant $t$ for our test system was a homothetic increase by a factor $r(t)$ of the load profile of the base case.

Figs. 7 and 8 represent the evolution of the suboptimality index, when assuming that all TSOs aim to minimize their active power losses or reactive power support, respectively. We note that, due to a nonoptimized initial state, the decentralized scheme achieves better performance after a certain delay, which depends on the memory factor. As observed in Table I for convergent cases, this delay is shorter when the memory factor is smaller. When TSOs aim to minimize active power losses, the decentralized control scheme achieves nearly-optimal performance, just like it did when applied to "time-invariant" systems. In this context, a small value of $\beta$ seems preferable since the greater time to convergence associated with high values produces a loss of performance. On the other hand, when TSOs aim 
TABLE V

Average CPU Times in Milliseconds ObTained With a CEnTRAlized OPTIMIZATION (COP), AND ONE ITERATION OF A DISTRIBUTED OPTIMIZATION (IDOP). THE TEST SYSTEM IS THE IEEE 118-BUS SYSTEM WITH THREE TSOS. PQ EQUIVALENTS ARE USED, With A MEMORY FACTOR $\beta=0.75$, AND A MEdium-Voltage Initial State

\begin{tabular}{|c||c||c||c|c|c|c|}
\hline$\gamma$ & COp & IDOp & TSO 1 & TSO 2 & TSO 3 & Load Flow \\
\hline 0 & 578 & 574 & 209 & 116 & 125 & 365 \\
\hline 1 & 6783 & 937 & 661 & 369 & 267 & 276 \\
\hline
\end{tabular}

to minimize reactive power support in their own areas, suboptimalities tend to be higher, even if they still tend to be acceptable with a carefully chosen value of $\beta$. We also notice here that, contrary to the previous example, decreasing too much the value of $\beta$ may considerably worsen the performance of the scheme.

\section{Computational Costs of THE Scheme}

As the decentralized control scheme based on PQ equivalents with a short-term memory factor achieves nearly optimal performance in the context of a time-invariant system, one could think of using this algorithm for distributed optimization of large-scale power systems. Indeed, as introduced in [20], paralleling OPF can lead to a significant decrease of computation time needed to centrally optimize such systems. Despite the small size of the illustrative benchmark systems, this section provides some numerical perspectives on the computational costs of solving an OPF in such a distributed way.

As emphasized in [21], the CPU time required for an OPF problem is strongly dependent on the mathematical formulation of the optimization function. Consequently, we analyze the computational costs corresponding to active power minimization and reactive power support minimization. The optimizations are run on a PC with Intel Core $2 \mathrm{~T} 72002.0 \mathrm{GHz}$ P2 processor and 2 GB memory. The optimization solver is MINOS in AMPL, which is described in [22] as being appropriate for small-scale power systems. For a centralized optimization, the reported CPU time is the time needed by MINOS in AMPL to solve the OPF. For a distributed optimization, the CPU times of each individual optimization are reported, and the "iteration CPU time" is defined as the sum of the maximal CPU time over the individual optimizations and the CPU time for the load flow that closes the iteration.

Table $\mathrm{V}$ reports the average CPU times obtained with a centralized optimization, and with one iteration of a distributed optimization based on PQ equivalents with $\beta=0.75$ and a medium-voltage initial state. The test system is the IEEE 118-bus system with three TSOs. As the CPU times are similar for a centralized scheme and one iteration of the distributed scheme applied to a minimization of active power losses, one could deduce that the scheme presents no interest for distributed optimization. However, the larger difference between the CPU times for minimization of reactive power support, which is more computationally challenging, shows that a distributed optimization based on single interconnection-based equivalent is potentially interesting. Indeed, the CPU time needed for one iteration being approximately seven times smaller than the time needed to solve the centralized problem, it could be of interest to use the distributed scheme in such a context, especially when few iterations lead to a nearly optimal solution.

\section{CONCLUSiOnS AND Future WORK}

We have addressed in this paper the problem of decentralized optimization of power systems. We have proposed a decentralized control scheme, where TSOs assume an external network equivalent for their neighboring areas at the interconnections and optimize at every iteration their control actions in a greedy way, i.e., without taking into consideration the impact that their actions may have on the other TSOs' objectives.

The optimization scheme does not require any explicit coordination and communication between the different TSOs. The communication is done implicitly, by measuring voltage and current values at interconnections, which depend on the actions taken by different TSOs. Those data are used further to fit the parameters of the equivalents. We have studied the performance of the control scheme for some particular equivalents and fitting procedures. We were particularly interested in identifying a suitable equivalent and its fitting procedure that lead to a good coordination of the TSO actions to achieve nearly optimal performance.

The study has mainly focused on what we have defined as single interconnection based equivalents, where a particular interconnection is modeled with a set of equality constraints between the voltage and the current at this interconnection. Also, the adopted fitting procedures estimate the parameters of the equivalents by using only local measurements at the interconnections. We considered several power system models, types of equivalents and memory factors for the fitting procedures. We analyzed through simulations the performance of the corresponding control scheme, and found out that it was strongly dependent on the functional form of the equality constraints and the memory factor. However, we have only been able to identify one pair of equivalent-memory factors, namely a PQ equivalent and a short-term memory factor, that was consistently performing well in the context of voltage optimization, regardless of the benchmark or the objective function used. More precisely, that pair was always leading to a decentralized optimization scheme that converged rapidly to a nearly optimal solution while, at the same time, ensuring a good compliance with the constraints.

This finding could lead to new rules for operating power systems in a decentralized fashion. For example, one could propose to incorporate this finding in the operation handbook of interconnected power system by proposing certain types of equivalents (and the corresponding procedures to fit their parameters) that should be used by the different TSOs to optimize the voltage management of their systems to minimize reactive power support within the network, active power losses, or a combination of both criteria.

There are, however, two main issues that should be investigated before using the outcome of this work as a guideline for approximately optimizing power systems in a decentralized way. The first concerns the obviously "time-invariant" nature of the power system considered in our simulation. As introduced in Section V, a detailed study of the control scheme should certainly be carried out when considering a power system, whose 
load/generation patterns or availability of different transmission elements is varying with time. In such a context, when the natural robustness of the decentralized control scheme is not sufficient for handling rapid changes in the operating conditions of the system, as in Fig. 8 for example, it would certainly be interesting to investigate adaptive methods for fitting the parameters of the equivalents.

The second issue concerns the assumption that all TSOs have the same objective function. Since it is not the case in practice, we should study whether the proposed scheme could perform well when the TSOs have different objective functions. One particular difficulty associated with this study is the definition of performance indices for decentralized control scheme, as one can not compare it with a single objective centralized control problem. Thus, the quantitative performance evaluation of the decentralized control scheme requires a well-established comparative framework for multi-party multi-objective optimizations. We may also question whether, even if the TSOs have the same type of objective function, a comparison with the results of a centralized control scheme is appropriate. Indeed, even if the scheme achieves nearly optimal performance according to this criterion, it may be unfair to some TSOs by penalizing heavily their own objective achievement. In such a context, and as emphasized in [23], it may be more relevant to associate the quality of the decentralized control scheme to its fairness than to its performance.

While those new issues may open new research directions, we also believe it would be interesting to investigate other aspects of this research: for example, to establish theoretical results proving, under appropriate assumptions, the convergence properties of the control scheme. We also suggest to set up methods to determine automatically good functional forms for equality constraints representing the equivalents. One way to address this problem would be to cast it as an optimization problem. The objective function would then use some of the criteria dealt with in this paper to assess the performance of the control schemes, and a search space composed of a large set of functional equality constraints (based on current and voltage measurements at the interconnections). As another possible research direction, we suggest to compare, using different points of view (simplicity, performance) the proposed decentralized control scheme with more advanced ones, based on certain types of explicit coordinations and/or communication between the TSOs.

\section{APPENDIX A}

\section{SINGLE-INTERCONNECTION BASED EQUIVALENTS AND FITTING PROCEDURES}

In this section, we detail the external network models used in our simulations and the procedure adopted to fit their parameters to the past observations.

We start the section by giving, for every of the considered single-interconnection based equivalents (PV, Thévenin-like, and $\mathrm{PQ}$ equivalents), the set of parametric equality constraints $\mathbf{E}_{\alpha}\left(I^{i, l}, V^{i, l}\right)=\mathbf{0}$ to which it corresponds. When writing the equality constraints, we will, for the sake of clarity, add the subscript $_{\alpha}$ to the parameters of those equalities.

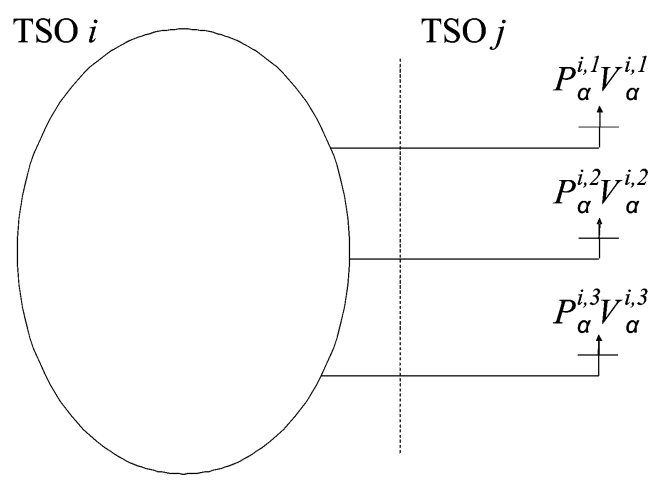

Fig. 9. TSO $i$ relying on PV equivalents to model a neighboring area controlled by TSO $j$ with which it has three interconnections.

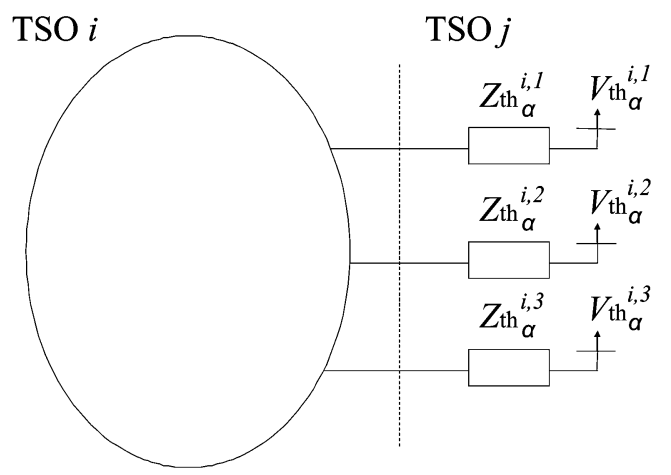

Fig. 10. TSO $i$ relying on Thévenin-like equivalents to model a neighboring area controlled by TSO $j$ with which it has three interconnections.

Afterwards, we describe the fitting procedure used in our simulations.

\section{A. PV Equivalent}

The PV equivalents are used to represent the power system areas beyond the interconnections as buses, whose voltage magnitudes and active power consumptions are constant. Fig. 9 illustrates in our multi-TSO context how a TSO uses those equivalents.

The set of parametric equality constraints $\mathbf{E}_{\alpha}\left(I^{i, l}, V^{i, l}\right)=\mathbf{0}$ corresponding to this equivalent can be written in the form ${ }^{2}$

$$
\begin{aligned}
\Re\left(V^{i, l} \cdot I^{i, l^{*}}\right)-P_{\alpha}^{i, l} & =0 \\
\left\|V^{i, l}\right\|-V_{\alpha}^{i, l} & =0 .
\end{aligned}
$$

Those equality constraints have two parameters, namely $P_{\alpha}^{i, l}$ and $V_{\alpha}^{i, l}$, which represent the active power and voltage magnitude related to the PV equivalent.

\section{B. Thévenin-Like Equivalent}

A Thévenin-like equivalent replaces the power system seen beyond an interconnection $l$ of a TSO $i$ by a bus, whose voltage phasor is constant, and by a line, whose impedance is also constant [24]. Fig. 10 illustrates, in our multi-TSO context, how a TSO uses those equivalents.

\footnotetext{
${ }^{2} a^{*}$ denotes the conjugate of the complex number $a$, and $\Re(a)$ its real part.
} 


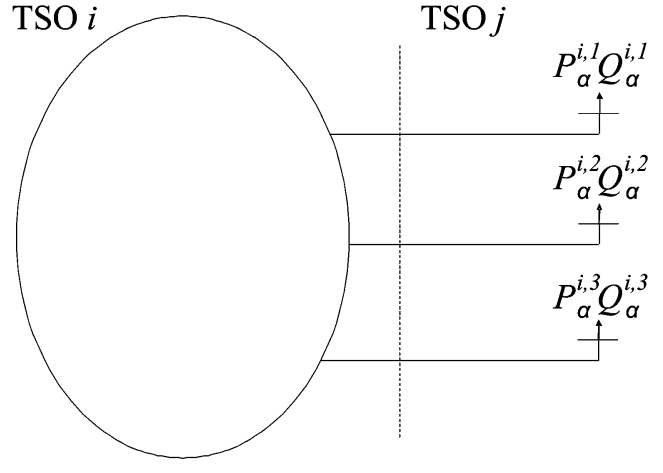

Fig. 11. TSO $i$ relying on PQ equivalents to model a neighboring area controlled by TSO $j$, with which it has three interconnections.

The set of parametric equality constraints $\mathbf{E}_{\alpha}\left(I^{i, l}, V^{i, l}\right)=\mathbf{0}$ corresponding to this equivalent can be written in the form ${ }^{3}$

$$
\begin{aligned}
& \Re\left(V^{i, l}-Z t h_{\alpha}^{i, l} \cdot I^{i, l}-V t h_{\alpha}^{i, l}\right)=0 \\
& \Im\left(V^{i, l}-Z t h_{\alpha}^{i, l} \cdot I^{i, l}-V t h_{\alpha}^{i, l}\right)=0
\end{aligned}
$$

where $V t h_{\alpha}^{i, l}$, and $Z t h_{\alpha}^{i, l}$ are the parameters of the Théveninlike equivalent. They represent the complex Thévenin-like voltage and the complex Thévenin-like impedance, respectively. We refer the equivalent as Thévenin-like because we apply the identification to a nonlinear system, which we consider as a time-varying sequence of linear system models.

\section{C. $P Q$ Equivalent}

A PQ equivalent replaces the power system beyond an interconnection $l$ of a TSO $i$ by a bus, whose active and reactive power consumptions are constant.

The set of parametric equality constraints $\mathbf{E}_{\alpha}\left(I^{i, l}, V^{i, l}\right)=\mathbf{0}$ corresponding to this equivalent can be written in the form

$$
\begin{aligned}
& \Re\left(V^{i, l} \cdot I^{i, l^{*}}\right)-P_{\alpha}^{i, l}=0 \\
& \Im\left(V^{i, l} \cdot I^{i, l^{*}}\right)-Q_{\alpha}^{i, l}=0 .
\end{aligned}
$$

The two parameters $P_{\alpha}^{i, l}$ and $Q_{\alpha}^{i, l}$ represent the active power and the reactive power consumption of the PQ equivalent.

\section{Fitting Procedure}

When considering single-interconnection based equivalents, the power system seen by a TSO $i$ beyond an interconnection $l$ is represented by the parametric equality constraints $\mathbf{E}_{\alpha}\left(I^{i, l}, V^{i, l}\right)=\mathbf{0}$. At instant $t$, the TSO $i$ fits the parameters of those equality constraints to the observations at interconnection $l$ to determine (in $\Omega$ ) the vector of parameters that fits best the past observations at this interconnection.

To determine what is the best fit in $\Omega$, we have first defined an index that assesses how good an element $\alpha_{A} \in \Omega$ is. This index is defined as follows

$$
-\sum_{k=0}^{t} \beta^{t-k}\left\|\mathbf{E}_{\alpha=\alpha_{A}}\left(\mathbf{H I}_{t}^{i, l}[k], \mathbf{H V}_{t}^{i, l}[k]\right)\right\|^{2}
$$

\footnotetext{
${ }^{3} \Im(a)$ denotes the imaginary part of the complex number $a$.
}

where $\beta \in[0,1]$ is the memory factor, defined in Section IV-C.1. By adopting such an index, we implicitly assume that the equivalent is better when the functional equality constraints are less violated (in terms of an Euclidean norm) when taking (as input) some observed values of the current and the voltage.

The function $\mathbf{F}$ adopted in our simulation determines in $\Omega$ the vector of parameters that maximizes this "goodness index"

$$
\begin{aligned}
& \mathbf{F}\left(\mathbf{H I}_{t}^{i, l}, \mathbf{H V}_{t}^{i, l}\right) \\
&=\underset{\alpha_{A} \in \Omega}{\arg \max }\left[-\sum_{k=0}^{t} \beta^{t-k} \| \mathbf{E}_{\alpha=\alpha_{A}}\right. \\
&\left.\quad \times\left(\mathbf{H I}_{t}^{i, l}[k], \mathbf{H V}_{t}^{i, l}[k]\right) \|\right] .
\end{aligned}
$$

Evaluation of this function requires solving a weighted leastsquares problem when $\mathrm{PV}, \mathrm{PQ}$, or Thévenin-like equivalents are considered. We found out that for specific types of equivalents, values of time $t$, and/or initial conditions, the optimization problem defined by (20) can have a nonunique solution. This is, for example, the case for a Thévenin-like equivalent, when $t=0$. We observed that this nonuniqueness of the solution sometimes makes our optimization software output arbitrarily large values of the parameters of the equivalents. A convenient way to circumvent this problem is to increase artificially the history of observations by padding it with several initial states. More specifically, we made $k$ vary from -2 to $t$ in (20), and chose values for voltages and currents at $t=-1$ and $t=-2$ those obtained by running a power flow algorithm after setting the generators' bus voltages at $99 \%$ and $101 \%$ of their true initial values at $t=0$, respectively.

\section{APPENDIX B \\ MORE ADVANCED EQUIVALENTS}

\section{A. REI Equivalent}

This section is dedicated to a description of the so-called REI equivalent. We assume in this paper that the TSO $i$ uses one single REI equivalent to represent the set of interconnections with another TSO $j$. Fig. 12 gives a physical representation of such equivalents in the context of a power system controlled by two TSOs, interconnected by three lines. As we can observe, a REI equivalent replaces each interconnection $l$ between TSO $i$ and TSO $j$ by an impedance $Z_{\alpha}^{l}$. Those impedances are connected to the same bus, which is itself connected through an impedance $Z_{\alpha}^{\mathrm{REI}}$ to a bus, whose voltage is $V_{\alpha}^{\mathrm{REI}}$. We refer the reader to [25] for a complement of information about this equivalent. While REI equivalent is less well known than PV, PQ, or Thévenin-like equivalents in power systems, its use has been reported in several research papers, such as [26], which addresses the problem of power flow scheduling in the context of noncoordinated decentralized control.

If $l_{1}^{(i, j)}, l_{2}^{(i, j)}, \ldots l_{n b I(i, j)}^{(i, j)}$ denote the interconnections between TSO $i$ and TSO $j$, then, the set of equality constraints corresponding to the power flow equations in the REI 


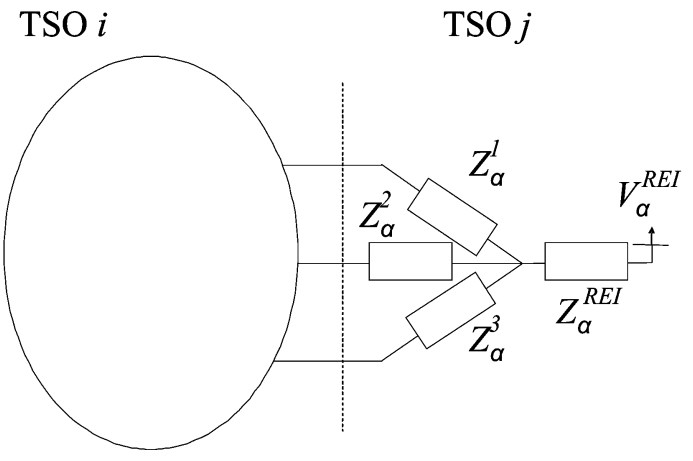

Fig. 12. TSO $i$ relying on a REI equivalent to model the power system seen beyond the three tie-lines shared with $\operatorname{TSO} j$.

equivalent network can be modeled by a generic expression $\mathbf{K}_{\alpha}^{(i, j)}\left(I^{i, l_{1}^{(i, j)}}, V^{i, l_{1}^{(i, j)}}, \ldots, I^{i, l_{n b I(i, j)}^{(i, j)}}, V^{i, l_{n b I(i, j)}^{(i, j)}}\right)=0$.

The parameters of the REI equivalent $\left(V_{\alpha}^{\mathrm{REI}}, Z_{\alpha}^{\mathrm{REI}}, Z_{\alpha}^{1}, Z_{\alpha}^{2}, \ldots, Z_{\alpha}^{n b I(i, j)}\right) \quad$ are $\quad$ fitted to past observations by using the same procedure as for single interconnection based equivalents. Similarly to (20), $\alpha$ is computed by maximizing a "goodness index," defined as in (19) by

$$
\begin{aligned}
&-\sum_{k=0}^{t} \beta^{t-k} \| \mathbf{K}_{\alpha=\alpha_{A}}^{n b I(i, j)}\left(\mathbf{H I}_{t}^{i, l_{1}^{(i, j)}}[k], \mathbf{H V}_{t}^{i, l_{1}^{(i, j)}}[k],\right. \\
&\left.\ldots, \mathbf{H I}_{t}^{i, l_{n b I(i, j)}^{(i, j)}}[k], \mathbf{H V}_{t}^{i, l_{n b I(i, j)}^{(i, j)}}[k]\right) \|^{2}
\end{aligned}
$$

\section{B. Non-Reduced Power System Equivalent}

The nonreduced power system equivalent, which is defined in [27], takes into consideration the entire network during local optimizations. The only thing unknown to a TSO $i$ when carrying an optimization at instant $t$, is the outcome of the optimization processes of the other TSOs, i.e., the actions $\mathbf{u}_{t+1}^{1}, \mathbf{u}_{t+1}^{2}, \ldots, \mathbf{u}_{t+1}^{n b T S O}$ that would be applied to the system at instant $t+1$. We suppose that in this case TSO $i$ predicts the actions of the other TSOs by computing a weighted average of their past control actions. More precisely, it predicts the control actions $\mathbf{u}_{j}$ that TSO $j$ will apply to the power system at the next instant by computing the following optimization problem:

$$
\begin{aligned}
& \mathbf{G}\left(\mathbf{H U}^{j}\right)= \\
& \underset{u^{j}}{\arg \max }\left[-\sum_{k=0}^{t} \beta^{t-k}\left\|u_{\alpha=\alpha_{A}}^{j}-\mathbf{H U}_{t}^{j}[k]\right\|^{2}\right]
\end{aligned}
$$

where $\mathbf{H U}_{t}^{j}[k]$ is the value of control settings of TSO $j$ at the instant $k$.

\section{REFERENCES}

[1] A. Adamson, A. Dessel, and L. Garver, "Generation reserve value of interconnections," IEEE Trans. Power App. Syst., vol. PAS-96, no. 2, pp. 337-346, 1977.

[2] J. Bialek, "Are blackouts contagious?," IEE Power Eng., vol. 17, pp. 10-13, Dec. 2003.

[3] L. Day, "Control area trends: Principles and responses," IEEE Comput. Appl. Power, vol. 8, pp. 34-39, Apr. 1995.
[4] Y. Li and V. Venkatasubramanian, "Coordination of transmission path transfers," IEEE Trans. Power Syst., vol. 19, no. 4, pp. 1607-1615, Aug. 2004.

[5] P. Panciatici, F. Bena, P. Pruvot, N. Janssens, J. Deuse, and M. Stubbe, "Centralized voltage control: A key point for optimal operation of power systems," in Cigre Paper 39-116, Paris, France, Sep. 1998.

[6] UCTE Operation Handbook, 2007. [Online]. Available: http://www. ucte.org/ohb/.

[7] N. Janssens, "Tertiary and secondary voltage control for the Belgian HV system," in Proc. IEE Colloq. Int. Practices in Reactive Power Control, London, U.K., Apr. 1993, pp. 81-84.

[8] H. Dommel and W. Tinney, "Optimal power flow solutions," IEEE Trans. Power App. Syst., vol. PAS-87, no. 10, pp. 1866-1876, Oct. 1968.

[9] N. Deeb and S. Shahidehpour, "Linear reactive power optimization in a large power network using the decomposition approach," IEEE Trans. Power Syst., vol. 5, no. 2, pp. 428-438, May 1990.

[10] F. Capitanescu, M. Glavic, D. Ernst, and L. Wehenkel, "Interior-point based algorithms for the solution of optimal power flow problems," Elect. Power Syst. Res., vol. 77, pp. 508-517, Apr. 2007.

[11] C. Taylor, "Reactive power today, best practices to prevent blackouts," IEEE Power and Energy Mag., vol. 4, pp. 104-102, Sep. 2006.

[12] B. Venkatesh, G. Sadasivam, and M. Khan, "A new optimal reactive power scheduling method for loss minimization and voltage stability margin maximization using successive multi-objective fuzzy LP technique," IEEE Trans. Power Syst., vol. 15, no. 2, pp. 844-851, May 2000.

[13] A. Marinakis, M. Glavic, and T. Van Cutsem, "Control of phase shifting transformers by multiple transmission system operators," in Proc. Power Tech 2007, Lausanne, Switzerland, Jul. 2007.

[14] M. Zima and D. Ernst, "On multi-area control in electric power systems," in Proc. PSCC 2005, Liege, Belgium, Aug. 2005.

[15] M. Ilic, E. Allen, J. Chapman, C. King, J. Lang, and E. Litvinov, "Preventing future blackouts by means of enhanced electric power systems control: From complexity to order," Proc. IEEE, vol. 93, no. 11, pp. 1920-1941, Nov. 2005.

[16] E. Miguelez, F. E. Cerezo, and L. Rodriguez, "On the assignment of voltage control ancillary service of generators in Spain," IEEE Trans. Power Syst., vol. 22, no. 1, pp. 367-375, Feb. 2007.

[17] G. Torres and V. Quintana, "An interior-point method for nonlinear optimal power flow using rectangular coordinates," IEEE Tran. Power Syst., vol. 13, no. 4, pp. 1211-1218, Nov. 1998.

[18] “Matpower User's Manual," R. Zimmerman, C. Murillo-Sanchez, and D. Gan, Eds., Sch. Elect. Eng., Cornell Univ., Ithaca, NY, 2005.

[19] H. Song, B. Lee, and Y. Moon, "Reactive optimal power flow incorporating margin enhancement constraints with non linear interior point method," Proc. Inst. Elect. Eng., Gen., Transm., Distrib., vol. 152, pp. 961-968, Nov. 2005.

[20] B. Kim and R. Baldick, "Coarse-grained distributed optimal power flow," IEEE Trans. Power Syst., vol. 12, no. 3, pp. 932-939, Aug. 1997.

[21] H. Wang, C. Murillo-Sanchez, R. Zimmerman, and R. Thomas, "On computational issues of market-based optimal power flow," IEEE Trans. Power Syst., vol. 22, no. 4, pp. 1185-1193, Nov. 2007.

[22] T. Orfanogianni and R. Bacher, "Increased OPF code development efficiency by integration of general purpose optimization and derivative computation tools," in Proc. 21 st IEEE Int. Conf. Power Industry Computer Applications (PICA'99), May 1999, pp. 123-129.

[23] Y. Phulpin, M. Begovic, M. Petit, and D. Ernst, "On the fairness of centralized decision-making strategies for multi-TSO power systems," in Proc. PSCC 2008, Glasgow, U.K., Jul. 2008.

[24] B. Milosevic and M. Begovic, "A network of phasor measurement units for voltage stability monitoring and control," IEEE Trans. Power Syst., vol. 18, no. 1, pp. 121-127, Feb. 2003.

[25] P. Dimo, Node Analysis of Power Systems. Manchester, U.K.: Abacus, 1975.

[26] M. Oatts, S. Erwin, and J. Hart, "Application of the REI equivalent for operations planning analysis of interchange schedules," IEEE Trans. Power Syst., vol. 5, no. 2, pp. 547-555, May 1990.

[27] L. Nepomuceno and A. Santos, Jr., "Equivalent optimization model for loss minimization: A suitable analysis approach," IEEE Trans. Power Syst., vol. 12, no. 4, pp. 1403-1412, Nov. 1997. 
Yannick Phulpin (S'06) received the electrical engineering degree in 2004 from Supélec, Paris, France, and from the Technical University of Darmstadt, Darmstadt, Germany. He is currently pursuing the Ph.D. degree in voltage stability and reactive power management at interconnections under the supervision of Prof. M. Begovic at the Georgia Institute of Technology, Atlanta.

$\mathrm{He}$ is an Assistant Professor at Supélec.

Miroslav Begovic (S'87-M'89-SM'92-F'04) is a Professor with the School of Electrical and Computer Engineering, Georgia Institute of Technology, Atlanta. His research interests are in the general area of computer applications in power system monitoring, protection and control, and design and analysis of renewable energy sources. He authored a section "System Protection" for the monograph The Electric Power Engineering Handbook (Boca Raton, FL: CRC, 2000).

Dr. Begovic was a Chair of the Working Group "Wide Area Protection and Emergency Control" and Vice-Chair of the Working Group "Voltage Collapse Mitigation" of the IEEE PES Power System Relaying Committee. He was a contributing member of the IEEE PES PSRC Working Group "Protective Aids to Voltage Stability", which received the IEEE Working Group Recognition Award in 1997. He is currently serving as Chair of the Emerging Technologies Coordinating Committee of the IEEE PES. He is a member of Sigma Xi, Tau Beta Pi, Eta Kappa Nu, and Phi Kappa Phi.
Marc Petit is a former student of the Ecole Normale Supérieure de Cachan, Paris, France. He received the Ph.D. degree from the University of Orsay, Orsay, France, in 2002.

Currently, he is an Associate Professor in the Department of Power and Energy Systems, Supélec, Paris, France.

Jean-Baptiste Heyberger received the M.Sc. degree in electrical and computer engineering from the University of Illinois at Urbana-Champaign in 2002 and the electrical engineering degree from Supélec, Paris, France, in 2003.

He is currently with RTE (the French TSO), Paris.

Damien Ernst (M'98) received the M.Sc. and Ph.D. degrees from the University of Liège, Liège, Belgium, in 1998 and 2003, respectively.

He is currently a Research Associate of the Belgian National Fund of Scientific Research (FNRS) and is affiliated with the Systems and Modeling Research Unit of the University of Liège. He spent the period 2003-2006 with the University of Liège as a Postdoctoral Researcher of the FNRS and held during this period positions as visiting researcher at CMU, MIT, and ETH. He spent the academic year 2006-2007 at Supélec, Paris, France, as a Professor. His main research interests are in the field of power system dynamics, optimal control, reinforcement learning, and design of dynamic treatment regimes. 